\title{
ERRATUM - revista Letras de Hoje edição v. 53, n. 4 (2018) - ID 33010 e ID 33161
}

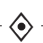

A Equipe Editorial da revista Letras de Hoje torna pública alterações na edição v. 53, n. 4, out.-dez. 2018, DOI: http://dx.doi.org/10.15448/1984-7726.2018.4: inclusão do nome do organizador LEONARDo TonUs no artigo "O contemporâneo é uma praça de guerra", com número de DOI: http://dx.doi.org/10.15448/1984-7726.2018.4.33010, publicado no periódico Letras de Hoje,

v. 53, n. 4, p. 459-460, out.-dez. 2018, assim como no artigo "Contemporaneity is a war zone", com número de DOI: http://dx.doi.org/10.15448/1984-7726.2018.4.33161, publicado no periódico Letras de Hoje, v. 53, n. 4, p. 461-462, out.-dez. 2018, conforme segue:

\section{Página 459}

Onde se lê: Ricardo Barberena

Pontifícia Universidade Católica do Rio Grande do Sul (PUCRS). Porto Alegre, Rio Grande do Sul, Brasil.

Leia-se: $\quad$ Ricardo Barberena ${ }^{1}$

Leonardo Tonus ${ }^{2}$

${ }^{1}$ Pontifícia Universidade Católica do Rio Grande do Sul (PUCRS). Porto Alegre, Rio Grande do Sul, Brasil.

${ }^{2}$ Sorbonne Université de Paris, Paris, França.

\section{Página 460}

Onde se lê: Barberena, $\mathrm{R}$.

Leia-se: $\quad$ Barberena, R., Tonus, L.

Onde se lê: Organizador

(1) RICARDO BARBERENA <ricardo.barberena@pucrs.br>

Pontifícia Universidade Católica do Rio Grande do Sul (PUCRS), Porto Alegre, Rio Grande do Sul, Brasil.

Leia-se: Organizadores

(D) RICARDO BARBERENA <ricardo.barberena@pucrs.br>

Pontifícia Universidade Católica do Rio Grande do Sul (PUCRS), Porto Alegre, Rio Grande do Sul, Brasil.

(D) LEONARDo TonUs<leotonusbr1@hotmail.com>

Sorbonne Université de Paris, Paris, França.

\section{Páginas 461}

Onde se lê: Ricardo Barberena

Pontifícia Universidade Católica do Rio Grande do Sul (PUCRS). Porto Alegre, Rio Grande do Sul, Brazil.

Leia-se: $\quad$ Ricardo Barberena ${ }^{1}$

Leonardo Tonus ${ }^{2}$

${ }^{1}$ Pontifícia Universidade Católica do Rio Grande do Sul (PUCRS). Porto Alegre, Rio Grande do Sul, Brazil.

${ }^{2}$ Sorbonne Université de Paris. Paris, France.

\section{Páginas 462}

Onde se lê: Barberena, $\mathrm{R}$.

Leia-se: $\quad$ Barberena, R., Tonus, L.

Onde se lê: Organizer

(D) RICARDO BARBERENA <ricardo.barberena@pucrs.br>

Pontifícia Universidade Católica do Rio Grande do Sul (PUCRS), Porto Alegre, Rio Grande do Sul, Brazil.

Leia-se: Organizers

(1) RICARDO BARBERENA <ricardo.barberena@pucrs.br>

Pontifícia Universidade Católica do Rio Grande do Sul (PUCRS), Porto Alegre, Rio Grande do Sul, Brazil.

(D) LeOnARDo TonUs <leotonusbr1@hotmail.com>

Sorbonne Université de Paris, Paris, France. 